\title{
Collateral benefits of studying the vagus nerve in bioelectronic medicine
}

Valentin A. Pavlov ${ }^{1,2}$ (D)

\begin{abstract}
Studies on the role of the vagus nerve in the regulation of immunity and inflammation have contributed to current preclinical and clinical efforts in bioelectronic medicine. In parallel, this research has generated new insights into the cellular and molecular mechanisms underlying the immunoregulatory functions of the vagus nerve within the inflammatory reflex. The vagus nerve and other cellular components of the inflammatory reflex are implicated in the regulation of bleeding, cancer, obesity, blood pressure, viral infections and other conditions. This collateral benefit broadens scientific horizons and provides new rationale for technological advances and therapeutic implications.
\end{abstract}

Keywords: Vagus nerve, Bioelectronic medicine, Cholinergic, Inflammation, Cytokines, Inflammatory diseases

\section{Background}

The vagus nerve has an essential role as a conduit of information between the brain and the periphery and in maintaining physiological homeostasis and defense mechanisms. During the last 20 years we have witnessed an explosion of new information substantially extending the knowledge about the regulatory functions of the vagus nerve. The recent discovery of the role of the vagus nerve in the regulation of immunity and inflammation within the inflammatory reflex is a major contribution to this new information (Borovikova et al., 2000; Tracey, 2002). Inflammation is a vital immune defense mechanism against invading pathogens, tissue damage and other immunological threats. Inflammation is tightly regulated to restore homeostasis (Nathan, 2002). However, inflammation does not always follow this normal scenario. Several forms of excessive, unresolved and chronic inflammation manifest and mediate pathogenesis in sepsis, rheumatoid arthritis, inflammatory bowel disease (IBD) and other inflammatory and autoimmune conditions (Nathan, 2002; Pavlov et al., 2018). Even obesity and cancer pathogenesis importantly involves immune dysregulation and aberrant inflammation (Pavlov \& Tracey, 2017; Balkwill \& Mantovani, 2001). Therefore,

Correspondence: vpavlov@northwell.edu

${ }^{1}$ Center for Biomedical Science and Bioelectronic Medicine, The Feinstein Institute for Medical Research, Northwell Health System, Manhasset, NY 11030, USA

${ }^{2}$ Donald and Barbara Zucker School of Medicine at Hofstra/Northwell, Hempstead, NY 11550, USA

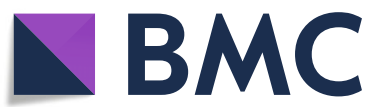

controlling inflammation is critically important in preventing and treating many conditions and diseases.

Recent studies demonstrated the important role of the vagus nerve in controlling pro-inflammatory cytokine release and inflammation within the inflammatory reflex (Tracey, 2002; Pavlov \& Tracey, 2017) (Fig. 1). The antiinflammatory and disease-alleviating efficacy of electrical vagus nerve stimulation (VNS) in numerous animal models of inflammatory disease have been described. This abundant knowledge provided a rationale for studying the therapeutic utility of bioelectronic VNS in human inflammatory and autoimmune diseases (Fig. 2). Recent successful clinical trials with implanted device-generated VNS in patients with rheumatoid arthritis, IBD and other conditions have validated the efficacy of this approach (Bonaz et al., 2016; Koopman et al., 2016). Both preclinical and clinical research on the anti-inflammatory function of the vagus nerve have contributed to current development in bioelectronic medicine (Fig. 2). This growing field utilizes new research insights into the regulatory functions of the nervous system and technological advances in the development of novel diagnostic and treatment approaches for a broad spectrum of diseases and conditions (Pavlov et al., 2018; Pavlov \& Tracey, 2019). In parallel with streamlining the studies on the anti-inflammatory functions of the vagus nerve in the context of bioelectronic medicine, considerable insights into the mechanisms underlying these functions have been generated. Moreover, the scope of disorders in which VNS or cholinergic modalities can be applied for

(C) The Author(s). 2019 Open Access This article is distributed under the terms of the Creative Commons Attribution 4.0 International License (http://creativecommons.org/licenses/by/4.0/), which permits unrestricted use, distribution, and reproduction in any medium, provided you give appropriate credit to the original author(s) and the source, provide a link to the Creative Commons license, and indicate if changes were made. The Creative Commons Public Domain Dedication waiver (http://creativecommons.org/publicdomain/zero/1.0/) applies to the data made available in this article, unless otherwise stated. 
therapeutic benefit has been extended. New discoveries related to the broader physiological role of cellular constituents of the vagus nerve-based inflammatory reflex have also been made. This research improves understanding of neural regulation, presents new therapeutic avenues both for bioelectronic medicine and other fields, leads to conceptual developments, and advances science as a whole. Here, I briefly summarize the role of the vagus nerve in the neuro-immune dialogue with relevance to bioelectronic medicine, and focus on the broader scope of new insights generated, designating them as collateral benefits.

\section{The vagus nerve: neurophysiological considerations}

The vagus nerve is a mixed nerve comprised of afferent (sensory, about $80 \%$ ) and efferent (motor, about 20\%) neurons. Vagus nerve afferent neurons are pseudounipolar cells residing in the nodose ganglia (Berthoud \& Neuhuber, 2000). The peripheral axonal terminals of these neurons in many visceral organs sense fluctuations in metabolic and cardiovascular homeostasis and this information is communicated to the brainstem nucleus tractus solitarius (NTS) where central axon endings terminate (Berthoud \& Neuhuber, 2000; Chavan et al., 2017; Pavlov \& Tracey, 2012). Neural communication between NTS and the dorsal motor nucleus of the vagus (DMN) mediates brainstem integration of afferent and efferent vagus nerve signaling, because DMN is a major source of efferent (motor) vagus neurons. These long preganglionic cholinergic neurons communicate with short postganglionic neurons in close range to or within the lungs, heart, pancreas and other innervated organs. Acetylcholine released from these neurons interacts with metabotropic muscarinic acetylcholine receptors (mAChRs) on smooth muscle cells, cardiac myocytes and glandular cells and regulates a broad spectrum of physiological functions including bronchoconstriction, heart rate, gastrointestinal motility and secretion and pancreatic endocrine and exocrine secretion (Pavlov \& Tracey, 2012).

\section{The vagus nerve in the neuro-immune dialogue: from preclinical studies to bioelectronic medicine}

Studies initiated about 20 years ago substantially broadened knowledge about the vagus nerve by revealing the role of this nerve in the regulation of immunity and inflammation (Borovikova et al., 2000; Tracey, 2002). This discovery complemented earlier studies demonstrating the role of afferent vagus nerve fibers in the gastrointestinal tract, hepatic portal system and other visceral organs in monitoring peripheral immune milieu (Chavan et al., 2017). These studies have demonstrated that afferent vagus neurons communicate signals about peripheral levels of IL-1 $\beta$, TNF and other inflammatory molecules to the brain and specifically to NTS (Fig. 1). Innovative research on the role of afferent and efferent vagus nerve

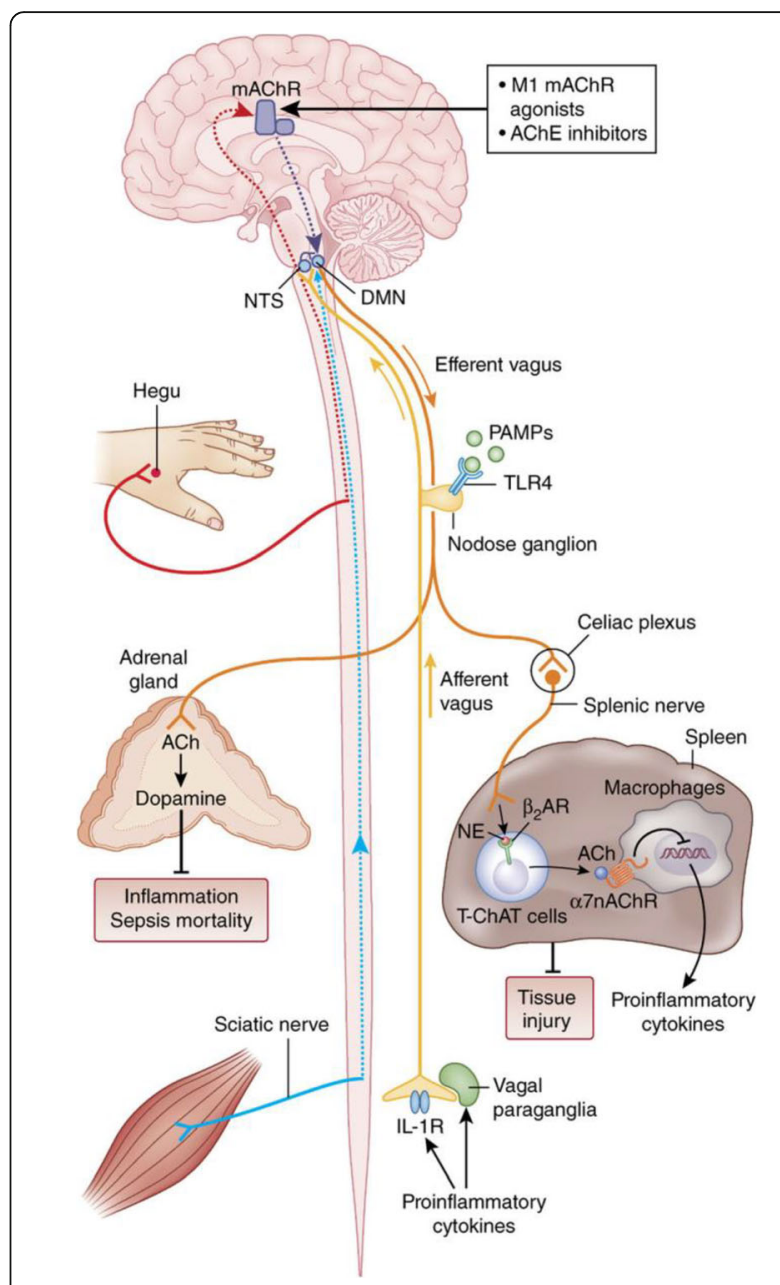

Fig. 1 The inflammatory reflex. In the inflammatory reflex, the activity of afferent vagus nerve fibers residing in the nodose ganglion is stimulated by cytokines and pathogen-associated molecular patterns (PAMPs). The signal is transmitted to the NTS. Reciprocal connections between the NTS and DMN mediate communication with and activation of efferent vagus nerve fibers from the DMN. The signal is propagated to the celiac ganglia and the superior mesenteric ganglion in the celiac plexus, where the splenic nerve originates. Norepinephrine (NE) released from the splenic nerve interacts with $\beta_{2}$-adrenergic receptors ( $\beta_{2}$-ARs) and causes the release of acetylcholine (ACh) from $T$ cells containing functional choline acetyltransferase (T-ChAT cells). ACh interacts with a7nAChRs on macrophages and suppresses proinflammatory cytokine release and inflammation. The inflammatory reflex can be activated through brain mAChR-mediated mechanisms by centrally-acting M1 mAChR agonists and acetylcholinesterase (AChE) inhibitors. Somatosensory activation by electroacupuncture at the Hegu point also causes activation of brain mAChR signaling, which then results in activation of efferent vagus and splenic anti-inflammatory signaling. Electroacupuncture at a different acupuncture point activates sciatic nerve signals, which by unknown mechanisms convert to efferent vagus nerve signaling to the adrenal medulla, resulting in dopamine release. Dopamine suppresses inflammation and improves survival in a model of sepsis. Vagus nerve and splenic nerve signaling mediated through a7nAChR on splenocytes controls inflammation in acute kidney injury and alleviates the condition. (Figure created by Debbie Maizels, Springer Nature, for Pavlov and Tracey 2017; reprinted, with permission, from the authors in conjunction with Springer Nature) 
signaling in the context of inflammation led to a major conceptual development - integrated afferent and efferent vagus nerve signaling regulates immune responses and inflammation within the inflammatory reflex (Tracey, 2002) (Fig. 1). Electrical vagus nerve stimulation (VNS) was used to discover the role of the efferent vagus nerve in controlling the levels of TNF and other pro-inflammatory cytokines (Pavlov \& Tracey, 2015). In addition, acetylcholine, a major mediator of efferent vagus nerve signaling, suppresses endotoxin-activated macrophage release of TNF, IL-1 $\beta$, and other pro-inflammatory cytokines (Borovikova et al., 2000). Numerous studies in rodent endotoxemia (Borovikova et al., 2000), sepsis (Huston et al., 2006), post-operative ileus (de Jonge et al., 2005), collageninduced arthritis (Levine et al., 2014), colitis (Meregnani et al., 2011), and other conditions have indicated that VNS can be used as a therapeutic approach to alleviate aberrant inflammation (Pavlov \& Tracey, 2015).

Insight from these ongoing pre-clinical studies recently led to the first clinical trials in patients with inflammatory disorders, including IBD (Crohn's disease) (Bonaz et al., 2016) and rheumatoid arthritis (Koopman et al., 2016). These preclinical and clinical studies accelerated the growing field of bioelectronic medicine (Pavlov et al., 2018; Pavlov \& Tracey, 2019) (Fig. 2). The first clinical trials utilized implanted devices for VNS that had already been clinically-approved for the treatment of epilepsy and depression (Bonaz, 2018). Encouraging results from the clinical trials generated parallel efforts focused on technological development, aimed at miniaturizing, improving the control and optimizing the therapeutic regimens of electrodes and devices (Levine et al., 2019). In parallel, development and testing of devices and approaches for non-invasive VNS in preand clinical settings and generating relevant mechanistic insight is also underway. For instance, non-invasive transcutaneous auricular VNS has been recently shown to activate NTS to DMN neural interactions and suppress inflammation in preclinical endotoxemia and postoperative ileus (Hong et al., 2018). In humans, the same type of stimulation has been shown to cause NTS and other brain neuronal activation patterns consistent with the "classical" afferent vagus nerve projections (Frangos et al., 2015). The therapeutic efficacy of transcutaneous auricular vagus nerve stimulation in depression (Kong et al., 2018) and other forms of non-invasive and invasive VNS in treating

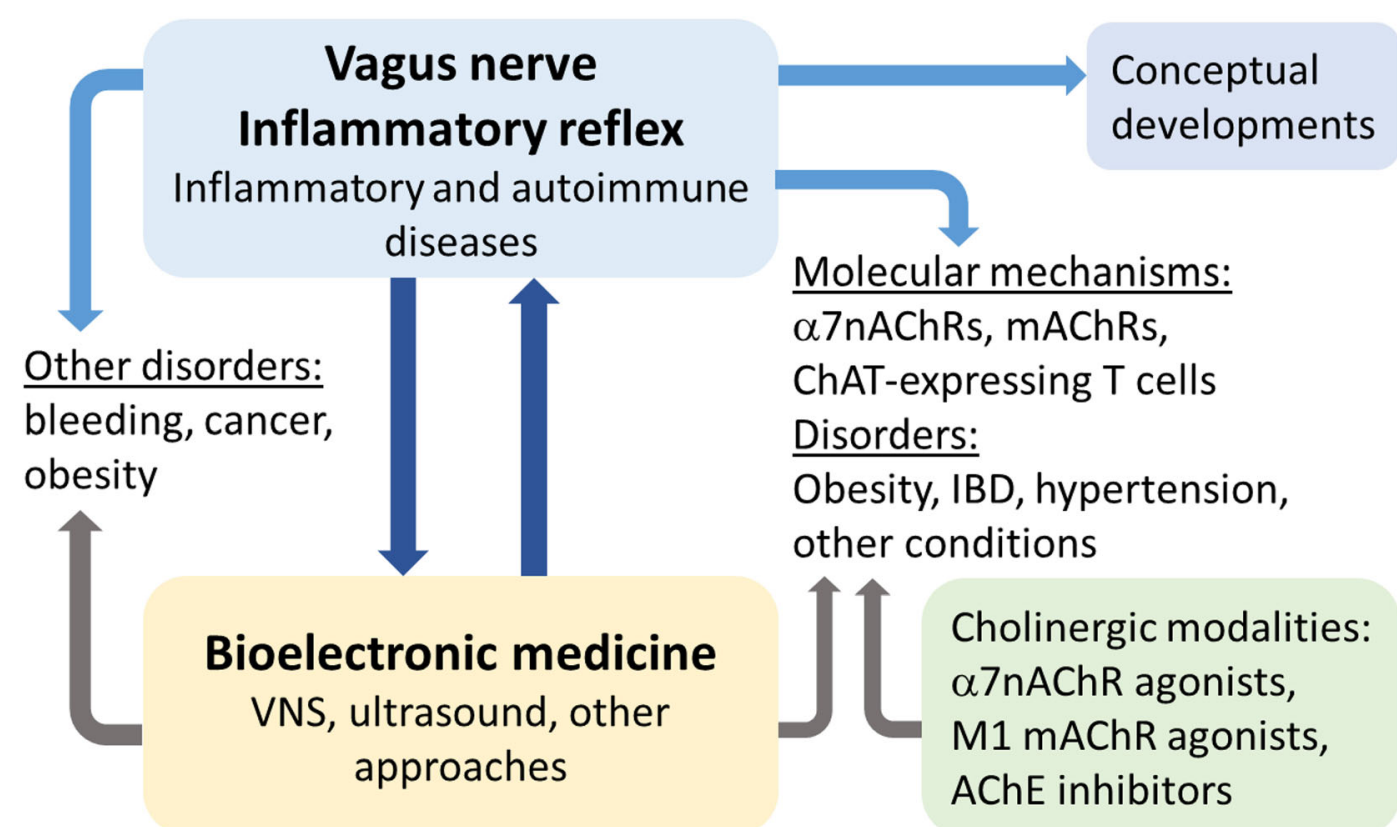

Fig. 2 The immunoregulatory functions of the vagus nerve in bioelectronic medicine and associated insights and advances. Preclinical studies on the immunoregulatory role of the vagus nerve and the inflammatory reflex in the context of inflammatory and autoimmune diseases have resulted in successful clinical trials exploring implanted device-generated VNS in Crohn's disease and rheumatoid arthritis under the umbrella of bioelectronic medicine. There is a symbiotic relationship between these and other ongoing clinical studies and active preclinical research. Studies stemming from this research have identified vagus nerve regulatory functions in bleeding, cancer, obesity and other disorders, which can also be targeted for therapeutic benefit by bioelectronic approaches. Molecular and cellular mechanisms underlying the inflammatory reflex involve a7nAChRs, brain mAChR and ChAT-expressing T lymphocytes. Their role as molecular therapeutic targets has been indicated in obesity-associated disorders, IBD (colitis), hypertensive and hypotensive conditions and other disorders, which can be targeted for therapeutic benefit by a7nAChR agonists, centrallyacting M1 mAChR agonists and AChE inhibitors and bioelectronics. All of this research, technological advances and therapeutic approaches have been accompanied by charting new relevant concepts and their ongoing validation (See text for details) 
chronic pain has been actively studied (Chakravarthy et al., 2015). In addition to VNS, the anti-inflammatory and therapeutic benefit of other approaches, including electrical acupuncture and ultrasound, activating neural circuitry within the inflammatory reflex was demonstrated in preclinical models of sepsis, acute kidney injury, and endotoxemia (Pavlov \& Tracey, 2017; Inoue et al., 2016; Torres-Rosas et al., 2014; Cotero et al., 2019). (Figs. 1, 2). In parallel, substantial insight into the cellular and molecular constituents of the inflammatory reflex considerably broadened the horizons for therapeutic developments.

\section{Revealing the mechanisms}

A somewhat surprising receptor mechanism underlies the anti-inflammatory role of the vagus nerve. It turned out that in contrast to many "classical" physiological functions, mediated through metabotropic mAChRs, the vagus nerve anti-inflammatory effects are mediated through ionotropic nicotinic acetylcholine receptors (nAChRs) (Wang et al., 2003). A specific role for a subset of these receptors, containing the $\alpha 7$ subunit and expressed on immune cells in this regulation (Figs. 1, 2) was revealed through several observations. VNS fails to significantly alter serum TNF levels in mice genetically deficient for the $\alpha 7 \mathrm{nAChR}$ during endotoxemia (Wang et al., 2003). In addition, while cholinergic treatment with nicotine significantly suppresses pro-inflammatory cytokine release by macrophages it fails to generate this effect in macrophages from $\alpha 7 \mathrm{nAChR} \mathrm{KO}$ mice or macrophages with $\alpha 7 \mathrm{nAChR}$ anti-sense downregulation (Wang et al., 2003). Furthermore, $\alpha 7 n A C h R$ expression in bone marrow-derived immune (non-T cells) was shown to play an essential role for the anti-inflammatory effect of VNS (Olofsson et al., 2012). A detailed understanding of intracellular mechanisms mediating $\alpha 7 \mathrm{nAChR}$-dependent cholinergic suppression of pro-inflammatory cytokine release has emerged. These mechanisms involve inhibition of the nuclear factor kappa-light-chain-enhancer of activated B cells (NF-kB) nuclear translocation (Guarini et al., 2003; Parrish et al., 2008), activation of the Janus kinase 2 - signal transducer and activator of transcription 3 (JAK2-STAT3) pathway (de Jonge et al., 2005), inhibition of the inflammasome (Lu et al., 2014), and inhibition of adenylyl cyclase 6 (Tarnawski et al., 2018).

The inflammatory reflex is also regulated centrally (in the brain) and several studies have revealed a role for mAChRs and specifically the M1 mAChR in this regulation in endotoxemia, inflammatory bowel disease (colitis), hemorrhagic shock and other conditions (Pavlov \& Tracey, 2015; Pavlov et al., 2006; Munyaka et al., 2014; Lee et al., 2010) (Fig. 1). Enhancing brain cholinergic signaling through administering centrally-acting acetylcholinesterase inhibitors such as galantamine also results in suppression of aberrant inflammatory responses through vagus nerve-mediated signaling in mouse models of endotoxemia, colitis and lupus (Pavlov et al., 2009; Ji et al., 2014; Pham et al., 2018). A very recent study utilizing selective optogenetic stimulation and advanced pharmacological approaches indicated a specific role for forebrain cholinergic signaling and M1 $\mathrm{mAChR}$ in the inhibition of peripheral inflammatory responses via vagus nerve signaling in murine endotoxemia (Lehner et al., 2019).

VNS results in suppression of splenic TNF, which has been identified as a substantial contributor to systemic TNF. How the vagus nerve controls cytokines in the spleen is noteworthy. The vagus nerve innervates the celiac ganglia and the superior mesenteric ganglion - a documented source of neurons within the splenic nerve (Pavlov \& Tracey, 2015). The endings of these catecholaminergic neurons release predominantly norepinephrine, which modulates $\mathrm{T}$ lymphocytes, which contain choline acetyltransferase (ChAT), the enzyme responsible for the synthesis of acetylcholine (Rosas-Ballina et al., 2011). These ChAT-expressing $\mathrm{CD}^{+}{ }^{+} \mathrm{T}$ lymphocytes play an important role in mediating the TNF-suppressing effect of the vagus nerve (Rosas-Ballina et al., 2011) (Figs. 1, 2). VNS increases splenic acetylcholine levels via splenic nerve catecholaminergic signaling acting on lymphocytes, and VNS fails to significantly suppress TNF levels in mice, lacking $\mathrm{T}$ cells (nude mice), during endotoxemia. In addition, passive adoptive transfer of ChAT-expressing T cells into nude mice restores the anti-TNF effect of VNS (Rosas-Ballina et al., 2011). Essential involvement of the vagus nerve-splenic nerve link has been indicated in neural regulation of immunity in endotoxemia (Rosas-Ballina et al., 2011), murine colitis (Munyaka et al., 2014; Ji et al., 2014), $\mathrm{T}$ cell activation and egress from the spleen, and the regulation of hypertension (Carnevale et al., 2016), renal ischemia-reperfusion injury (Inoue et al., 2016), and other conditions (Pavlov \& Tracey, 2015).

\section{The collateral scientific and therapeutic benefit}

The intriguing role of ChAT-expressing $\mathrm{T}$ cells as a source of acetylcholine in the neural circuitry of the inflammatory reflex importantly implicated these cells in the regulation of innate immunity and inflammation. This discovery generated new research efforts that provided insights extending the scope of ChAT-expressing $\mathrm{T}$ cell functional repertoire in physiological and disease contexts. The physiological regulation of blood pressure is complex and involves several brain-orchestrated and peripheral neuro-endocrine and metabolic components. Acetylcholine is importantly involved in blood pressure regulation and the vasorelaxant properties of this 
molecule related to the release of nitric oxide are well documented. However, the source of acetylcholine in this context has remained somewhat enigmatic. Further characterization of the distinct T-cell population defined by ChAT identified them as $\mathrm{CD} 4{ }^{+} \mathrm{CD} 44^{\text {hi }} \mathrm{CD} 62 \mathrm{~L}^{\text {lo }} \mathrm{T}$ helper cells by gene expression (Olofsson et al., 2016). Mice with selective deficiency of ChAT expression in $\mathrm{CD}^{+}{ }^{+}$cells have higher arterial blood pressure as compared to littermate control mice, and infusion of Jurkat $\mathrm{T}$ cells overexpressing ChAT significantly lowers blood pressure (Olofsson et al., 2016). In addition, co-incubation of these Jurkat $\mathrm{T}$ cells and endothelial cells increases phosphorylation of endothelial nitric oxide synthase, and nitrate and nitrite levels in conditioned media, suggesting activated release of nitric oxide - a molecule with a key role in vasolelaxation (Olofsson et al., 2016). These findings demonstrate the important role of $\mathrm{CD}_{4}^{+} \mathrm{T}_{\mathrm{ChAT}}$ cells in blood pressure regulation. They provide a rationale for further studies on these cells in hypotension and hypertension, and designing new therapeutic approaches focused on cell-mediated vasorelaxation (Olofsson et al., 2016) (Fig. 2). Another very recent study revealed the important role of lymphocyte-released acetylcholine in the regulation of chronic viral infections. Lymphocytic choriomeningitis virus (LCMV) infection is associated with significantly increased ChAT levels in both $\mathrm{CD}_{4}^{+}$and $\mathrm{CD}^{+} \mathrm{T}$ cells in an IL-21-dependent manner (Cox et al., 2019). Mice with selective Chat deficiency within the $T$ cell compartment have impaired vasodilation in response to infection, decreased migration of antiviral $\mathrm{T}$ cells into infected tissues, and substantially compromised control of chronic LCMV clone 13 infection. These findings represent a genetic proof of function for ChAT in T cells during viral infection and implicate ChAT-expressing $\mathrm{T}$ cells in antiviral immunity (Cox et al., 2019).

The growing insight into the inflammatory reflex and the receptor mechanisms mediating efferent vagus nerve anti-inflammatory output prompted new clinicallyoriented research. The anti-inflammatory and diseasealleviating effects of many $\alpha 7 \mathrm{nAChR}$ agonists, including nicotine, GTS-21, choline, and PNU-282987 have been demonstrated in preclinical settings of sepsis, postoperative neuroinflammation, ischemia-reperfusion injury, obesity and type 2 diabetes, and other disorders (Parrish et al., 2008; Pavlov et al., 2007; Chatterjee et al., 2017; Mavropoulos et al., 2017; Terrando et al., 2011; Wang et al., 2011; Li et al., 2013; Yeboah et al., 2008; Hoover, 2017; Terrando \& Pavlov, 2018) (Fig. 2). Centrally-acting $\mathrm{mAChR}$ agonists and acetylcholinesterase inhibitors have shown promising efficacy in murine models of sepsis, inflammatory bowel disease, obesity, lupus, and other conditions (Munyaka et al., 2014; Ji et al., 2014; Pham et al., 2018; Rosas-Ballina et al., 2015; Satapathy et al., 2011; Yang \& Yang, 2018; Hanes et al., 2015) (Fig. 2). One of these centrally-acting acetylcholinesterase inhibitors - galantamine is a clinically-approved drug for the treatment of Alzheimer's disease. This fact, the abundant information about the safety profile of this drug and the rationale from preclinical studies (Satapathy et al., 2011) facilitated performing a recent clinical trial with this drug in patients with the metabolic syndrome (Consolim-Colombo et al., 2017). In this randomized placebo-controlled trial, treatment with clinically-approved doses of galantamine significantly alleviated the inflammatory state and insulin resistance and modulated the autonomic neural function towards vagus nerve predominance (Consolim-Colombo et al., 2017).

Another line of research emerging from studying the role of the vagus nerve in immune regulation identified a new role of this nerve in the regulation of bleeding (hemorrhage) (Czura et al., 2010) (Fig. 2). VNS in pigs subjected to ear resection significantly shortens the bleeding time and increases the thrombin/antithrombin III complex formations (Czura et al., 2010). These findings suggest that VNS can be further explored for therapeutic benefit in hemorrhage-related conditions.

An accumulating body of data also reveals an intriguing role for the vagus nerve in preclinical scenarios of cancer, including breast, gastric and pancreatic cancer (Chavan et al., 2017; Pavlov \& Tracey, 2015) (Fig. 2). Two recent studies provide important insights into the role of the vagus nerve and cholinergic signaling in pancreatic exocrine cancer. In mice with pancreatic ductal adenocarcinoma, subdiaphragmatic vagotomy results in increased tumor growth and worsened survival (Partecke et al., 2017). This is associated with increased TNF levels and increased number of tumor associated macrophages. In addition, the survival of TNF knockout mice implanted with the tumor is significantly longer (Partecke et al., 2017). In another study subdiaphragmatic vagotomy in mice with LSL-Kras ${ }^{+/ G 12 D}$; Pdx1-Cre model of this cancer significantly accelerates tumorigenesis, whereas activation of cholinergic signaling via administration of a mAChR agonist has suppressive effects (Renz et al., 2018). Stimulated cholinergic signaling specifically results in suppression of the cancer stem cell compartment, CD11b ${ }^{+}$ myeloid cells, TNF levels, and metastasis in the liver (Renz et al., 2018). These findings suggest the potential of exploring VNS and cholinergic modalities in the treatment of such an aggressive disease with notoriously vague symptoms as pancreatic exocrine cancer (Fig. 2).

Chronic low-grade inflammation has a documented role in promoting insulin resistance and other metabolic derangements in obesity and obesity-related conditions, including the metabolic syndrome, type 2 diabetes, cardiovascular disease and non-alcoholic steatohepatitis (Pavlov \& Tracey, 2012). There is a growing interest in exploring vagus nerve circuitry by using bioelectronics 
in the treatment of the modern pandemics of obesity and obesity-related disorders by exploring vagus nerve efferent anti-inflammatory signaling and vagus nerve afferents with a role in satiety (Pavlov \& Tracey, 2012; Yao et al., 2018) (Fig. 2).

Research on the immunomodulatory function of the vagus nerve and its brain regulation also contributed significantly to formulating the concepts of the immunological homunculus (Pavlov et al., 2018; Tracey, 2007), the set point function of the immune responses (Tracey, 2009), the neuroimmune communicatome (Olofsson et al., 2017; Metz \& Pavlov, 2018), and innate immune exhaustion in sepsis survivors (Zaghloul et al., 2017) (Fig. 2). Ongoing and future studies utilizing advanced technics for molecular mapping and selective modulation of neural circuitry will undoubtedly provide new insight within the framework of these conceptual models and indicate new therapeutic avenues (Pavlov \& Tracey, 2017; Chang et al., 2015; Chang, 2019).

\section{Conclusions}

Improved understanding of the role of the vagus nerve in controlling inflammation and innovative applications of this knowledge led to current clinical developments in treating inflammatory and autoimmune diseases with implantable bioelectronics. These advances benefited parallel technological developments related to improving these devices and testing new devices for non-invasive bioelectronic VNS (Levine et al., 2019; Mertens et al., 2018). In parallel, other approaches within the scope of bioelectronic medicine, including electrical acupuncture and ultrasound for activating circuitries in the inflammatory reflex have showed promising therapeutic efficacy (Fig. 2). Demonstrating a role for the vagus nerve in the control of bleeding, cancer, and obesity, and the important involvement of a subset of ChAT-expressing $\mathrm{T}$ cells in the regulation of blood pressure and very recently - in viral infection are all important examples of discoveries stemming from studying the immunoregulatory role of the vagus nerve. Revealing the molecular mechanisms of efferent vagus nerve anti-inflammatory control have indicated the therapeutic applicability of $\alpha 7 \mathrm{nAChR}$ agonists and centrally-acting acetylcholinesterase inhibitors, with first examples of clinical validation in clinical settings (Fig. 2). These developments represent a broad spectrum of collateral benefit of innovative research on the vagus nerve with current clinical implications in bioelectronic medicine (Fig. 2). Multidisciplinary collaborative research efforts will continue to advance bioelectronic medicine and benefit other disciplines with the goal of creating therapeutic approaches that will make a difference for our patients.

\section{Abbreviations}

ACh: Acetylcholine; AChE: Acetylcholinesterase; ChAT: Choline

acetyltransferase; DMN: Dorsal motor nucleus of the vagus; IBD: Inflammatory bowel disease; IL-1: 1ßinterleukin-1ß; IL-21: Interleukin-21; JAK2-STAT3: Janus kinase 2- signal transducer and activator of transcription 3; LCMV: Lymphocytic choriomeningitis virus; mAChRs: Muscarinic acetylcholine receptors; nAChRs: Nicotinic acetylcholine receptors; NE: Norepinephrine; NF-kB: Nuclear factor kappa-light-chain-enhancer of activated B cells; NTS: Nucleus tractus solitarius; PAMPs: Pathogen-associated molecular patterns; TNF: Tumor necrosis factor; VNS: Vagus nerve stimulation; $\beta_{2}$-ARs: $\beta_{2}$-adrenergic receptors

\section{Acknowledgements}

The author thank Kevin J. Tracey and Christine N. Metz for their comments on this paper.

\section{Funding}

The research of V.A.P. with relevance to this paper is supported by grants R01GM121102 and R01GM128008 from the National Institutes of Health, National Institute of General Medical Sciences.

Availability of data and materials

Not applicable.

Authors' contributions

The author wrote the paper. The author read and approved the final manuscript.

Ethics approval and consent to participate

Not applicable.

\section{Consent for publication}

Not applicable.

\section{Competing interests}

V.A.P. is an author of patents broadly related to the topic of this paper and has assigned his rights to the Feinstein Institute for Medical Research. V.A.P. is also an executive editor of the journal Bioelectronic Medicine.

\section{Publisher's Note}

Springer Nature remains neutral with regard to jurisdictional claims in published maps and institutional affiliations.

Received: 29 March 2019 Accepted: 24 April 2019

Published online: 16 May 2019

References

Balkwill F, Mantovani A. Inflammation and cancer: back to Virchow? Lancet (London, England). 2001;357(9255):539-45.

Berthoud HR, Neuhuber WL. Functional and chemical anatomy of the afferent vagal system. Auton Neurosci. 2000;85(1-3):1-17.

Bonaz B. Is-there a place for vagus nerve stimulation in inflammatory bowel diseases? Bioelectronic Med. 2018;4(1):4.

Bonaz B, Sinniger V, Hoffmann D, Clarencon D, Mathieu N, Dantzer C, et al. Chronic vagus nerve stimulation in Crohn's disease: a 6-month follow-up pilot study. Neurogastroenterol Motil. 2016;28(6):948-53.

Borovikova LV, Ivanova S, Zhang M, Yang H, Botchkina Gl, Watkins LR, et al. Vagus nerve stimulation attenuates the systemic inflammatory response to endotoxin. Nature. 2000;405(6785):458-62.

Carnevale D, Perrotta M, Pallante F, Fardella V, lacobucci R, Fardella S, et al. A cholinergic-sympathetic pathway primes immunity in hypertension and mediates brain-to-spleen communication. Nat Commun. 2016;7:13035

Chakravarthy K, Chaudhry H, Williams K, Christo PJ. Review of the uses of vagal nerve stimulation in chronic pain management. Curr Pain Headache Rep. 2015;19(12):54.

Chang RB. Optogenetic control of the peripheral nervous system. Cold Spring Harb Perspect Med. 2019. https://doi.org/10.1101/cshperspect.a034397.

Chang RB, Strochlic DE, Williams EK, Umans BD, Liberles SD. Vagal sensory neuron subtypes that differentially control breathing. Cell. 2015;161(3):622-33.

Chatterjee PK, Yeboah MM, Solanki MH, Kumar G, Xue X, Pavlov VA, et al. Activation of the cholinergic anti-inflammatory pathway by GTS-21 
attenuates cisplatin-induced acute kidney injury in mice. PLoS One. 2017; 12(11):e0188797.

Chavan SS, Pavlov VA, Tracey KJ. Mechanisms and therapeutic relevance of neuro-immune communication. Immunity. 2017;46(6):927-42.

Consolim-Colombo FM, Sangaleti CT, Costa FO, Morais TL, Lopes HF, Motta JM, et al. Galantamine alleviates inflammation and insulin resistance in patients with metabolic syndrome in a randomized trial. JCl insight. 2017;2(14).

Cotero V, Fan Y, Tsaava T, Kressel AM, Hancu I, Fitzgerald P, et al. Noninvasive sub-organ ultrasound stimulation for targeted neuromodulation. Nat Commun. 2019;10(1):952.

Cox MA, Duncan GS, Lin GHY, Steinberg BE, Yu LX, Brenner D, et al. Choline acetyltransferase-expressing $T$ cells are required to control chronic viral infection. Science (New York, NY). 2019;363(6427):639-44.

Czura CJ, Schultz A, Kaipel M, Khadem A, Huston JM, Pavlov VA, et al. Vagus nerve stimulation regulates hemostasis in swine. Shock (Augusta, Ga). 2010;33(6):608-13.

de Jonge WJ, van der Zanden EP, The FO, Bijlsma MF, van Westerloo DJ, Bennink RJ, et al. Stimulation of the vagus nerve attenuates macrophage activation by activating the Jak2-STAT3 signaling pathway. Nat Immunol. 2005;6(8):844-51.

Frangos E, Ellrich J, Komisaruk BR. Non-invasive access to the Vagus nerve central projections via electrical stimulation of the external ear: fMRl evidence in humans. Brain stimulation. 2015;8(3):624-36.

Guarini S, Altavilla D, Cainazzo MM, Giuliani D, Bigiani A, Marini H, et al. Efferent vagal fibre stimulation blunts nuclear factor-kappaB activation and protects against hypovolemic hemorrhagic shock. Circulation. 2003;107(8):1189-94.

Hanes WM, Olofsson PS, Kwan K, Hudson LK, Chavan SS, Pavlov VA, et al. Galantamine attenuates type 1 diabetes and inhibits anti-insulin antibodies in non-obese diabetic mice. Mol Med. 2015;21(1):702-8.

Hong GS, Zillekens A, Schneiker B, Pantelis D, de Jonge WJ, Schaefer N, et al. Non-invasive transcutaneous auricular vagus nerve stimulation prevents postoperative ileus and endotoxemia in mice. Neurogastroenterol Motil. 2018;31:e13501. https://doi.org/10.1111/nmo.13501

Hoover DB. Cholinergic modulation of the immune system presents new approaches for treating inflammation. Pharmacol Ther. 2017;179:1-16.

Huston JM, Ochani M, Rosas-Ballina M, Liao H, Ochani K, Pavlov VA, et al. Splenectomy inactivates the cholinergic antiinflammatory pathway during lethal endotoxemia and polymicrobial sepsis. J Exp Med. 2006;203(7):1623-8.

Inoue T, Abe C, Sung SS, Moscalu S, Jankowski J, Huang L, et al. Vagus nerve stimulation mediates protection from kidney ischemia-reperfusion injury through alpha7nAChR+ splenocytes. J Clin Invest. 2016;126(5):1939-52.

Ji H, Rabbi MF, Labis B, Pavlov VA, Tracey KJ, Ghia JE. Central cholinergic activation of a vagus nerve-to-spleen circuit alleviates experimental colitis. Mucosal Immunol. 2014;7(2):335-47.

Kong J, Fang J, Park J, Li S, Rong P. Treating depression with transcutaneous auricular Vagus nerve stimulation: state of the art and future perspectives. Frontiers in psychiatry. 2018;9:20.

Koopman FA, Chavan SS, Miljko S, Grazio S, Sokolovic S, Schuurman PR, et al. Vagus nerve stimulation inhibits cytokine production and attenuates disease severity in rheumatoid arthritis. Proc Natl Acad Sci U S A. 2016;113(29):8284-9.

Lee ST, Chu K, Jung KH, Kang KM, Kim JH, Bahn JJ, et al. Cholinergic anti-inflammatory pathway in intracerebral hemorrhage. Brain Res. 2010;1309:164-71.

Lehner KR, Silverman HA, Addorisio ME, Roy A, Al-Onaizi MA, Levine Y, et al. Forebrain Cholinergic Signaling Regulates Innate Immune Responses and Inflammation. Frontiers in immunology. 2019;10:585.

Levine YA, Faltys M, Chernoff D. Harnessing the inflammatory reflex for the treatment of inflammation-mediated diseases. Cold Spring Harb Perspect Med. 2019

Levine YA, Koopman FA, Faltys M, Caravaca A, Bendele A, Zitnik R, et al. Neurostimulation of the cholinergic anti-inflammatory pathway ameliorates disease in rat collagen-induced arthritis. PLoS One. 2014;9(8):e104530.

Li F, Chen Z, Pan Q, Fu S, Lin F, Ren H, et al. The protective effect of PNU-282987, a selective alpha7 nicotinic acetylcholine receptor agonist, on the hepatic ischemia-reperfusion injury is associated with the inhibition of high-mobility group box 1 protein expression and nuclear factor kappaB activation in mice. Shock (Augusta, Ga). 2013;39(2):197-203.

Lu B, Kwan K, Levine YA, Olofsson PS, Yang H, Li J, et al. alpha7 nicotinic acetylcholine receptor signaling inhibits inflammasome activation by preventing mitochondrial DNA release. Mol Med. 2014;20:350-8.

Mavropoulos SA, Khan NS, Levy ACJ, Faliks BT, Sison CP, Pavlov VA, et al. Nicotinic acetylcholine receptor-mediated protection of the rat heart exposed to ischemia reperfusion. Mol Med. 2017;23.
Meregnani J, Clarencon D, Vivier M, Peinnequin A, Mouret C, Sinniger V, et al. Anti-inflammatory effect of vagus nerve stimulation in a rat model of inflammatory bowel disease. Auton Neurosci. 2011;160(1-2):82-9.

Mertens A, Raedt R, Gadeyne S, Carrette E, Boon P, Vonck K. Recent advances in devices for vagus nerve stimulation. Expert Rev Med Devices. 2018;15(8):527-39.

Metz CN, Pavlov VA. Vagus nerve cholinergic circuitry to the liver and the gastrointestinal tract in the neuroimmune communicatome. Am J Physiol Gastrointest Liver Physiol. 2018.

Munyaka P, Rabbi MF, Pavlov VA, Tracey KJ, Khafipour E, Ghia JE. Central muscarinic cholinergic activation alters interaction between splenic dendritic cell and CD4+CD25- T cells in experimental colitis. PLoS One. 2014:9(10):e109272.

Nathan C. Points of control in inflammation. Nature. 2002;420(6917):846-52.

Olofsson PS, Katz DA, Rosas-Ballina M, Levine YA, Ochani M, Valdes-Ferrer SI, et al. alpha7 nicotinic acetylcholine receptor (alpha7nAChR) expression in bone marrow-derived non-T cells is required for the inflammatory reflex. Mol Med. 2012;18:539-43.

Olofsson PS, Metz CN, Pavlov VA. The Neuroimmune Communicatome in Inflammation In: Cavaillon J, Singer M, editors. Inflammation: from molecular and cellular mechanisms to the clinic, vol. 4: Wiley-VCH Verlag GmbH \& Co. KGaA; 2017. p. 1485-516.

Olofsson PS, Steinberg BE, Sobbi R, Cox MA, Ahmed MN, Oswald M, et al. Blood pressure regulation by $\mathrm{CD} 4(+)$ lymphocytes expressing choline acetyltransferase. Nat Biotechnol. 2016;34(10):1066-71.

Parrish WR, Rosas-Ballina M, Gallowitsch-Puerta M, Ochani M, Ochani K, Yang LH, et al. Modulation of TNF release by choline requires alpha7 subunit nicotinic acetylcholine receptor-mediated signaling. Mol Med. 2008;14(9-10):567-74.

Partecke LI, Kading A, Trung DN, Diedrich S, Sendler M, Weiss F, et al. Subdiaphragmatic vagotomy promotes tumor growth and reduces survival via TNFalpha in a murine pancreatic cancer model. Oncotarget. 2017:8(14):22501-12.

Pavlov VA, Chavan SS, Tracey KJ. Molecular and functional neuroscience in immunity. Annu Rev Immunol. 2018;36:783-812.

Pavlov VA, Ochani M, Gallowitsch-Puerta M, Ochani K, Huston JM, Czura CJ, et al. Central muscarinic cholinergic regulation of the systemic inflammatory response during endotoxemia. Proc Natl Acad Sci U S A. 2006;103(13):5219-23.

Pavlov VA, Ochani M, Yang LH, Gallowitsch-Puerta M, Ochani K, Lin X, et al. Selective alpha7-nicotinic acetylcholine receptor agonist GTS-21 improves survival in murine endotoxemia and severe sepsis. Crit Care Med. 2007;35(4):1139-44.

Pavlov VA, Parrish WR, Rosas-Ballina M, Ochani M, Puerta M, Ochani K, et al. Brain acetylcholinesterase activity controls systemic cytokine levels through the cholinergic anti-inflammatory pathway. Brain Behav Immun. 2009;23(1):41-5.

Pavlov VA, Tracey KJ. The vagus nerve and the inflammatory reflex-linking immunity and metabolism. Nat Rev Endocrinol. 2012;8(12):743-54.

Pavlov VA, Tracey KJ. Neural circuitry and immunity. Immunol Res. 2015; 63(1-3):38-57.

Pavlov VA, Tracey KJ. Neural regulation of immunity: molecular mechanisms and clinical translation. Nat Neurosci. 2017;20(2):156-66.

Pavlov VA, Tracey KJ. Bioelectronic medicine: updates, challenges and paths forward. Bioelectronic Medicine. 2019:5(1):1.

Pham GS, Wang LA, Mathis KW. Pharmacological potentiation of the efferent Vagus nerve attenuates blood pressure and renal injury in a murine model of systemic lupus erythematosus. Am J Physiol Regul Integr Comp Physiol. 2018.

Renz BW, Tanaka T, Sunagawa M, Takahashi R, Jiang Z, Macchini M, et al. Cholinergic signaling via muscarinic receptors directly and indirectly suppresses pancreatic tumorigenesis and Cancer Stemness. Cancer Dis. 2018:8(11):1458-73.

Rosas-Ballina M, Olofsson PS, Ochani M, Valdes-Ferrer SI, Levine YA, Reardon C, et al. Acetylcholine-synthesizing T cells relay neural signals in a vagus nerve circuit. Science (New York, NY). 2011;334(6052):98-101.

Rosas-Ballina M, Valdes-Ferrer SI, Dancho ME, Ochani M, Katz D, Cheng KF, et al. Xanomeline suppresses excessive pro-inflammatory cytokine responses through neural signal-mediated pathways and improves survival in lethal inflammation. Brain Behav Immun. 2015:44:19-27.

Satapathy SK, Ochani M, Dancho M, Hudson LK, Rosas-Ballina M, Valdes-Ferrer SI, et al. Galantamine alleviates inflammation and other obesity-associated complications in high-fat diet-fed mice. Mol Med. 2011;17(7-8):599-606. 
Tarnawski L, Reardon C, Caravaca AS, Rosas-Ballina M, Tusche MW, Drake AR, et al. Adenylyl cyclase 6 mediates inhibition of TNF in the inflammatory reflex. Front Immunol. 2018;9:2648.

Terrando N, Eriksson LI, Ryu JK, Yang T, Monaco C, Feldmann M, et al. Resolving postoperative neuroinflammation and cognitive decline. Ann Neurol. 2011;70(6):986-95.

Terrando N, Pavlov VA. Editorial: neuro-immune interactions in inflammation and autoimmunity. Front Immunol. 2018;9:772.

Torres-Rosas R, Yehia G, Pena G, Mishra P, del Rocio Thompson-Bonilla M, Moreno-Eutimio MA, et al. Dopamine mediates vagal modulation of the immune system by electroacupuncture. Nat Med. 2014;20(3):291-5.

Tracey KJ. The inflammatory reflex. Nature. 2002;420(6917):853-9.

Tracey KJ. Physiology and immunology of the cholinergic antiinflammatory pathway. J Clin Invest. 2007;117(2):289-96.

Tracey KJ. Reflex control of immunity. Nat Rev Immunol. 2009:9(6):418-28.

Wang H, Yu M, Ochani M, Amella CA, Tanovic M, Susarla S, et al. Nicotinic acetylcholine receptor alpha7 subunit is an essential regulator of inflammation. Nature. 2003;421(6921):384-8.

Wang X, Yang Z, Xue B, Shi H. Activation of the cholinergic antiinflammatory pathway ameliorates obesity-induced inflammation and insulin resistance. Endocrinology. 2011;152(3):836-46.

Yang YPY, Yang J. Galantamine protects against hydrochloric acid aspirationinduced acute respiratory distress syndrome in rabbits. Trop J Pharm Res. 2018;17 (4):669-673.

Yao G, Kang L, Li J, Long Y, Wei H, Ferreira CA, et al. Effective weight control via an implanted self-powered vagus nerve stimulation device. Nat Commun. 2018;9(1):5349

Yeboah MM, Xue X, Duan B, Ochani M, Tracey KJ, Susin M, et al. Cholinergic agonists attenuate renal ischemia-reperfusion injury in rats. Kidney Int 2008;74(1):62-9.

Zaghloul N, Addorisio ME, Silverman HA, Patel HL, Valdes-Ferrer SI, Ayasolla KR, et al. Forebrain cholinergic dysfunction and systemic and brain inflammation in murine Sepsis survivors. Front Immunol. 2017:8:1673.

Ready to submit your research? Choose BMC and benefit from:

- fast, convenient online submission

- thorough peer review by experienced researchers in your field

- rapid publication on acceptance

- support for research data, including large and complex data types

- gold Open Access which fosters wider collaboration and increased citations

- maximum visibility for your research: over $100 \mathrm{M}$ website views per year

At $\mathrm{BMC}$, research is always in progress.

Learn more biomedcentral.com/submissions 